\title{
IMPORTANCE OF PROPER BREATHING IN SINGING
}

\author{
Rajeshwari Devi ${ }^{1} \bowtie$ \\ Research Scholar, Department of Dance \& Music, Manipur University, Kanchipuram, India
}

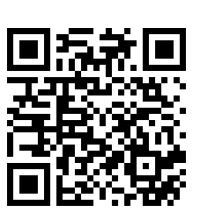

Research Scholar, Department of Dance \& Music, Manipur University, Kanchipuram, India

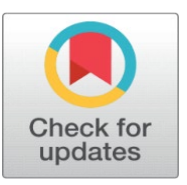

Received 01 September 2021

Accepted 25 September 2021

Published 20 October 2021

\section{CorrespondingAuthor \\ Kh. Rajeshwari Devi, m_thoibasingh@yahoo.com \\ DOI}

10.29121/shodhkosh.v2.i2.2021.39

Funding: This research received no specific grant from any funding agency in the public, commercial, or not-for-profit sectors.

Copyright: (C) 2021 The Author(s). This is an open access article distributed under the terms of the Creative Commons Attribution License, which permits unrestricted use, distribution, and reproduction in any medium, provided the original author and source are credited.

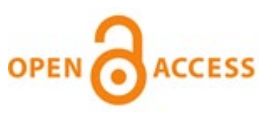

\section{ABSTRACT}

There is much truth in the statement that 'breathing is singing' proper breathing is essential for singing and learning of breath correctly is actually one of the most important aspects to improve the singing voice. Improving breathing technique allows a singer to have more control over their voice and enable them to explore a broader range of notes and to have greater capacity of air and it gives control to expel the breath in order to sustain on the notes for a longer period. The intensity of the voice depends upon the breath forces which regulate the amplitude of the vibrations of the vocal chords. If the foundation of breathing technique is not proper a singer will have poor results, and later will suffer. Good breath management skills are necessary if a singer hopes to be able to sing great wilt skill. So, breathing exercise are critical for any aspiring vocalist to achieve singing success. This paper try to discuss on the Important of proper breathing in singing.

Keywords: Breathing, Control, Singer, Vocal Cord, Voice, Diaphragm

\section{INTRODUCTION}

Voice is a universal of music. The human voice may be regarded as the first musical instrument which cannot be compared with any other one thing. Mankind was gifted will voice before the invention of instruments. Human voice is superior to all man-made instruments, because it does not have a definite range and the range of this instrument is extended through practice. No inventor of today has built a musical instrument in exact imitation of the insensitive flexible vocal tract with its elastic and marvelously movable walls. Human voice consists of four parts a motor, a vibrator, a resonator, and an articulator. Some of the musical instruments have the first three parts, in some forms but the articulator is the characteristic of the human voice.

1) The motor, consisting respiratory muscles and lungs.

2) The vibrator, consisting vocal chords.

3) The resonator, consists of throat mouth and the nasal and head cavities

4) The articulator consisting of tongue, lips, teeth, and palate.

These parts seem to a variety of size and proportion and moreover variable according to the will or feeling of the individual. A variety in tone quality is produced by this sensitivity which constitutes a modifying power. There are two system of voice training methods- empirical and scientific. The first method takes into account the quality of sound produced and empirically determines the methods which would make the sound better. These methods are based upon trial-and-error process. Secondly, the scientific method analysis 
the cause of tonal impurity and advised to acquire scientific action to produce a good tone and to erase the tonal impurity

Singing requires a delicate control of the three sets of muscles those of inspiration and expiration, those of phonation and those articulation. Inspiration is the phase of ventilation in which air expelled from the lungs. These two processes are involved in respiration. During inspiration the diaphragm is drawn down by contraction and there is the relaxation of the intercostal muscles. The abdomen muscles move downward to make room for the depressed diaphragm, which causes a vacuum in the chest. The lungs expand to fill the vacuum and the air rushes in to fill the expanding lungs. In expiration the diaphragm and the external intercostal muscle are relaxed, thus lessening the depth of the thorax. This pressure forces the air from the lungs and prepares them for another inspiration.

Diaphragm is a large, dome shaped which is in the form like an inverted bowl located at the base of the lungs. It is attached by a strong tend on to the spinal column behind and to the lowest part of the walls of the thorax. It forms the floor of the thorax and the roof of the abdomen. Diaphragmatic breathing or abdominal breathing is correct or natural for both male and female. Breathing from the diaphragm is beneficial for everyone, it encourages full oxygen exchange. Breathing deeply with full control with the whole body from below not with the upper chest only is important for every people. And every teacher and student should remember that singing requires wind and muscle, so breathing power must be fully developed.

Breath control is necessary for performers. It affects the range, volume, and speed of singing. Power and largeness of tone depend upon the right use of the resonant cavities and the volume of breath used under proper control. Full control of breath insures success to a good voice, and without it, the best voice lead to downfall.

\section{PRELIMINARY SUGGESTION OF BREATHING EXERCISE}

1) Proper breathing exercises to be energize and purifying demand plenty of fresh air.

2) Practicing only ten minutes at a time, three times a day is better for the beginners.

3) The best time for practicing is in the morning; with the window open practicing directly after a meal is harmful for everyone.

4) Gently lengthens the time without overdoing.

5) Breathe as deeply and broadly as possible.

6) Use the complete breath, not only the high breath or mid breath.

7) Keep the mouth shut, when not singing, speaking, or practicing.

Additionally, learning to take a deep breathing exercise will be able to improve overall vocal quality, proper posture and attitude are also important aspects of improving the sound of voice. Breathing exercise can improve one's breathing performance which ultimately improves the singing voice as well. Discovering correct breathing exercise for singing is definitely a requirement for any aspiring vocalist. 


\section{DEEP BREATH AND BREATH CONTROL}

1) Inhale deeply through the nose hold the breath for some seconds then exhale the breath through the mouth.

2) Inhale the breath very slowly and steadily through the smallest possible opening of the lips, then exhale the air at once through the open mouth.

3) Inhale as in 1. Through the nose and hold the breath for some seconds and exhale through nostril slowly. This practice must be done with an open throat and depend on the breathing muscles for control of exhaling air.

4) Inhale the breath through the smallest opening of the lips as in two hold it a few seconds, then expel very slowly through the smallest possible of mouth.

5) Inhale through the more very slowly and hold the air a few seconds, purse the lips tightly and exhale the air through them very slowly and steadily until the exhalation complete.

6) Vocalised 'Ah' on an easy pitch and assist on the note for a longer period until you feel strained. After vocalizing 'hum' on the same note and attempt to sustain on it for a longer period.

Natural level or suitable level of pitch is important for each individual. The laryngeal muscle of the singer makes strained by using unsuited pitch level either too higher too low. The level of pitch is based upon the physical characteristics of individual laryngeal mechanism.

Laryngeal mechanisms are an important parameter of voice production. It is part of respiratory system and serves many biological functions. It is a cartilaginous framework connected by ligaments and muscles. They are directly related to the notion of registers and may be defined as different vocal qualities or changes of timbre by the singers. It consists of the following cartilages, they are:

1. Epiglottis 2. Thyroid cartilage 3. Cricoid cartilage 4. Arytenoid cartilage 5. Corniculate cartilages and 6. Cuneiform cartilage.

Singers and scientists all over the world are agreed, that the style of breathing determines human voice quality to a great extent, voice is a very delicate and sympathetic instrument. Hence, proper, or correct breathing habits should be maintained welly, from the early years itself, because the early incorrect training may damage the voice completely or may impairs the voice quality badly as to require of long time to regain good quality. The most effective methods of voice production for any individuals, professional singers' students and teachers pursuing all types and styles of singing may gain the complete solution from voice training. There are many scientific aspects of vocal training which is related to voice cultivation and the practice of technically developed exercise. This process of exercising method will help the singers to analyse their own voice level and to operate the vocal apparatus for the perfect voice modulation. Although vocal mechanism cannot be changed due to genetically determined features, the right techniques and methods can mould and make an individual voice more melodious. An ideal and a good singer should be able to manipulate all the types and styles of music. The concept of an ideal voice differs according to the different systems of music, the techniques and methods of voce training are also different. The scientific methods and principles of voice production have carefully studied and experimented practically as voice training or voice culture is intimately bound up with the systems of music. Every nation has established their own voice \& culture methods for the artistic purpose of singing and the requisites of the particular style 
of music through the ages. There are four main types of singing for both male and female voice. Tenor and bass are for men, and soprano and Alto for woman. There are many more categories between these like mezzo- soprano, contralto, countertenor, and baritone which are applied to many singers.

There are a number of varieties of vocal techniques that can help to develop and improve in all round vocal performance. The lifestyle of an individual will also affect the ability of singing, damaging habits will also surely exhibit from achieving potential in singing.

\section{COMMON AND IMPORTANT DIRECTIONS}

1) Coming down the scale and produce the lowest note audible in the voice.

2) To pronounce the word 'hung' at once and prolong the tone, not on the vowel sound but on the 'ng' sound.

3) 3.Passing to 'ee' in a careful way from 'ng' without changing the initial focus of the sensation of nasal and head resonance.

4) Passing 'ee' to 'oo' and from 'oo' to 'oh' with the least possible movement of lips and chin.

5) The sound of each vowel must be distinct in enunciation and pure. Give each of them true individuality without blurring one with the other.

Here are some more things in common which can be done to keep the vocal technique in proper condition.

Never sing at full volume without fully warming up the voice and speak at a normal volume.

Eat a healthy and balanced diet and drink plenty of water to stay hydrated.

Practice for daily in a right way, and get trained will a qualified teacher regularly

\section{CONCLUSION}

Proper breath management techniques are imperative when discussing the pathway to healthy sustainable singing. Every music system has its own demand in terms of voice production and styles and has training methods in accordance with its type of music. So, it can be said that there is no prioritize type of ideal voice as each system of music is variedly constituted to the ingredients which make up its originality.

Human voice is the only living instrument of music. It is the most delicate and beautiful instrument which requires very careful handling. Incorrect use of vocal mechanism always leads to vocal impurities. Scientific methods of vocal training provide different methods and techniques suggest remedies to for tonal impurities. It is up to us to preserve and cultivate this Devine instrument given by the Almighty to humanity.

\section{REFERENCES}

Fillebrown, T. (2006). Resonance in Singing and Speaking (3rd ed.). Oliver Ditson Company. 


\section{Rajeshwari Devi}

Lewis, L. (2021, August 25). The singers workshop -Breathe Control.

Durga, S. A. K. (1978). Voice Culture : With Special reference to South Indian Music. Journal of the Indian Musicological Society. Indian Musicological Society.

Unnikrishnan, T. (2017). The Singing Voice : (Practical \&Technical Aspects of Voice Culture For All Styles). Independently Published. 